\title{
$1 \quad$ Warming sensitivity of spring phenology of deciduous species lies in their bud energy budget
}

Affiliations :

$4{ }^{1}$ Computational and Applied Vegetation Ecology - CAVElab, Department of Environment, Faculty of

5 Bioscience Engineering, Ghent University, Gent, Belgium

$6 \quad{ }^{2}$ CSIC, Global Ecology Unit CREAF-CSIC-UAB, 08193 Bellaterra, Catalonia, Spain

$7 \quad{ }^{3}$ CREAF, 08193 Cerdanyola del Vallès, Catalonia, Spain

*Correspondence to: marc.peaucelle@ugent.be

e-mail addresses: marc.peaucelle@ugent.be,josep.penuelas@uab.cat,hans.verbeeck@ugent.be

Keywords: plant phenology, budburst, temperature, light, energy budget, modelling, climate warming

Type of article: Perspective

Abstract: 132 words

Number of words: Main text - 2201 words

24 Number of references: 62

Number of figures: 3 
29 Spring phenology is mainly driven by temperature in extratropical ecosystems. Contrasting 30 responses of foliar phenology to climatic warming, however, have been reported in recent decades, 31 raising important questions about the role of other environmental constraints, especially light. In 32 fact, temperatures differ substantially between plant tissues and the air because plants absorb and 33 lose energy. Yet, phenology studies always substitute plant tissue temperature by air temperature. 34 Here, we explored how solar radiation, wind, and bud traits might affect spring phenology of 35 deciduous forests through the energy budget of buds. We show that air temperature might be an 36 imprecise and biased predictor of bud temperature. Our current interpretation of the plant 37 phenological response to warming should be reconsidered, which will require new observations of bud traits and temperature for accurately quantifying their energy budget. 
Plant phenology, the study of the timing of life-cycle events, drives several ecosystem functions, such as plant productivity and biomass, but also local and global climates by affecting biogeochemical and biogeophysical processes, such as carbon storage and energy fluxes ${ }^{1,2}$, and the abundance and diversity of local flora and fauna, such as pollinators and herbivores ${ }^{3,4}$. Understanding the environmental controls and responses of plant phenology to climate change is thus essential for several sectors, e.g. agriculture, forestry and gardening ${ }^{5}$, but also for conservation ${ }^{6}$ and public health ${ }^{7}$ (e.g. allergies).

\section{Foliar phenology and temperature}

Leaves control plant water loss and carbon assimilation and are thus central to the growth of other plant

organs. Temperature is one of the main drivers of foliar phenology in extratropical ecosystems ${ }^{8}$. Cold

the beginning of the season. Temperature during the growing season will control foliar development, carbon assimilation, transpiration, plant growth and the establishment of new buds for the following year.

Finally, cold temperatures at the end of the growing season are sensed by plants as a signal for foliar senescence 9 .

Climatic warming has strongly shifted phenophases in the Northern Hemisphere in recent decades $^{1,10-12}$. Rising temperatures have lengthened the annual growth cycle by advancing leaf unfolding in spring and delaying leaf fall in autumn ${ }^{13}$, albeit with variations among species ${ }^{14}$ and regions ${ }^{15}$. Recent evidence, though, suggests that the sensitivity of spring phenology to warming is decreasing in northern forests ${ }^{16}$ and that the rate of change in plant productivity does not match that of air temperature ${ }^{17}$. Indeed, plant phenology may be acclimated to long-term biogeographical constraints ${ }^{18-20}$ and may be co-limited by several other factors, such as light $^{21}$, water ${ }^{9,22,23}$ and nutrients ${ }^{24}$. These observations suggests that warming does not have the same effect everywhere ${ }^{25}$, which has increased interest in other environmental drivers in recent decades, especially illustrated by multiple debates about the specific role of light (and photoperiodism) in spring phenology $\mathrm{y}^{21,26-33}$.

\section{The controversial effect of light: different definitions}

How light affects spring phenology remains an open question. Most commonly, its effect is considered via photoperiod, often referred to as daylength. The daylength hypothesis implies that the quality and/or quantity of light is somehow directly sensed by plants through biochemical mechanisms. Some recent studies suggest that the spectral composition of light can indeed influence foliar phenology ${ }^{34,35}$. Light also plays a key role in regulating phytohormones, but the underlying mechanisms 
treated as the sum of insolation over a specific period ${ }^{36}$, for which plants need a specific quotum for a phenological event to occur. The quantity and quality of light depend on plant location, which is the main reason why a response to daylength has often been proposed as a safety mechanism against frost at high latitudes and elevations. Only 35\% of the woody species in the Northern Hemisphere, however, depend on daylength as a direct signal for leaf-out ${ }^{21}$, and these species are mainly at mid- to low latitudes

Light effect on spring phenology is still being debated. Recent studies nonetheless suggest a complex interaction between temperature and light. Daytime and nighttime temperatures during winter and spring have an asymmetrical effect on leaf unfolding ${ }^{37-41}$, with a greater weight of temperature during the day ${ }^{38,42,43}$. Whether or not plants are able to sense light, radiation has a physical impact on plants: it affects the temperatures of their tissues. We will use the example of budburst in the following arguments to illustrate that omitting this radiation effect introduces large biases into the interpretation of spring phenological responses based on air temperature.

\section{The forgotten effects of radiation and wind}

Bud temperature $\left(\mathrm{T}_{\text {bud }}\right)$ depends on its energy balance ${ }^{44}$. During the day, plant tissues absorb both shortwave (SW, visible and near-infrared) and longwave (LW, infrared) radiation from the sky but also radiation emitted and reflected by the surrounding environment (vegetation, soil) (Figure 1a). Only a fraction ( $\alpha$, absorptivity) of SW radiation will be absorbed depending on bud traits such as color, coating, shape and size (Figure 1b), while most LW radiation will be absorbed by buds. According to the StefanBoltzmann law, buds lose energy via LW radiation emission, while they absorb LW radiation emitted from surrounding objects. Finally, a part of their energy is lost by conduction and mostly by convection $^{45,46}$ (e.g. due to wind) while leaves lose an important part of their energy via transpiration.

$\mathrm{T}_{\text {bud }}$ increases when energy gains exceed losses (Figure $2 \mathrm{a}$ ) and vice-versa. $\mathrm{T}_{\text {bud }}$ can thus be lower than air temperatures $\left(\mathrm{T}_{\text {air }}\right)$ on clear nights ${ }^{45}$ or because of wind. On the other hand, $\mathrm{T}_{\text {bud }}$ can be significantly higher than $\mathrm{T}_{\text {air }}$ during the day. The link between $\mathrm{T}_{\text {bud }}$ and energy balance has been known for more than 30 years ${ }^{44,45}$. Since then, all major studies linking temperature and photoperiod to phenological changes, however, have not accounted for the true temperature of plant organs. 
a.

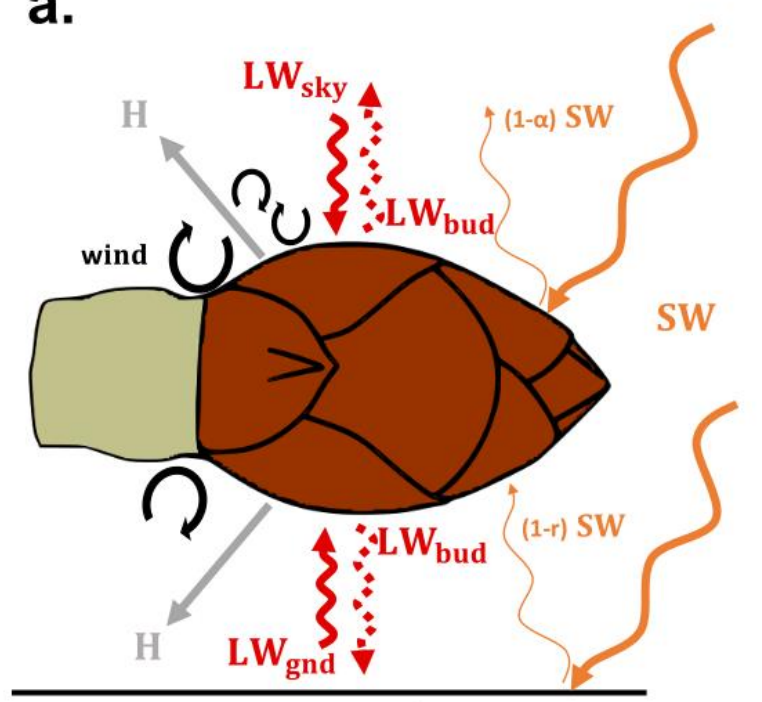

Ground b.

\section{Bud Traits}

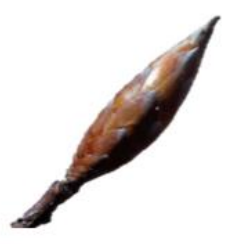

Color

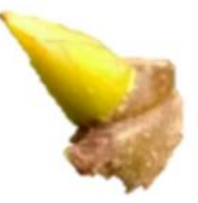

Shape Coating Size
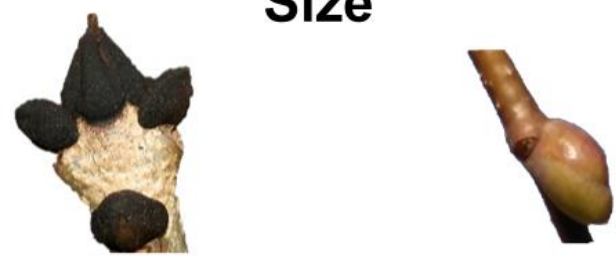

Figure 1 | Energy budget of buds and bud traits. a. Buds lose energy through convection and conduction $(\mathrm{H})$. Buds absorb incoming shortwave (visible and near-infrared, SW) and longwave (infrared, LW) radiation from the sky ( $\mathrm{LW}_{\text {sky }}$ ) and the surrounding environment (here simplified as $\mathrm{LW}$ radiation from the ground, $\mathrm{LW}_{\mathrm{gnd}}$ ). Buds emit $\mathrm{LW}$ radiation as a function of their temperature ( $\left(\mathrm{LW}_{\text {bud }}\right)$. Only a fraction $(\alpha)$ of SW radiation is absorbed by buds, depending on the properties of their surfaces. Buds also absorb a small fraction of SW reflected from the ground (1-r). b. Illustration of bud traits influencing solar absorptivity, heat conduction and convection processes, and hence, bud temperature.
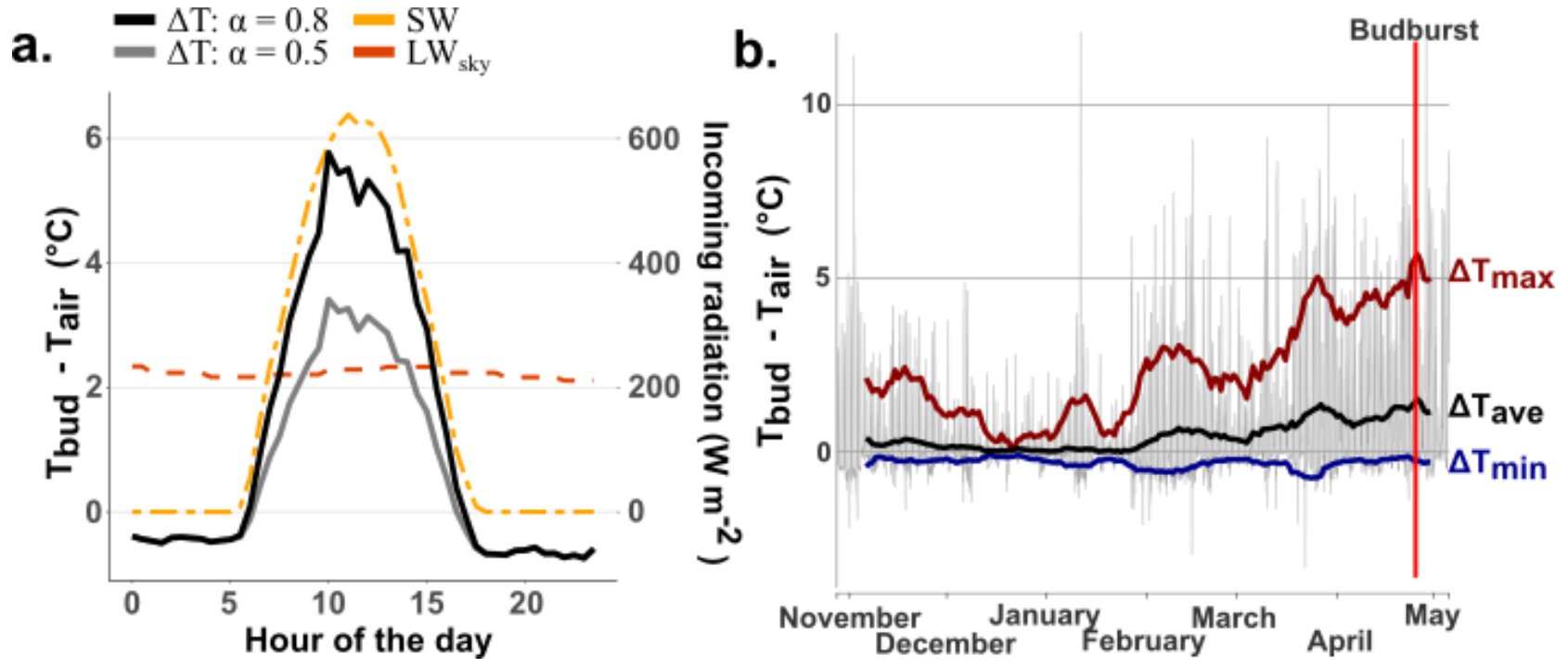

Figure 2 | Simulated differences in temperature between buds and the air $(\Delta T)$ from energy balance. a. Daily variation in $\Delta \mathrm{T}$ for an exposed bud and a typical day in April for two solar absorptivities: $\alpha=0.5$ (typical for broadleaves ${ }^{47}$ ) and $\alpha=0.8$ (typical for needle leaves ${ }^{47}$ ). b Example of $\Delta \mathrm{T}$ simulated under idealized conditions (e.g. exposed buds in a deciduous canopy) using meteorological observations for winter and spring collected at the Hesse FLUXNET site ${ }^{48}$ (Beech forest, France). The grey lines represent half-hourly differences in temperature simulated using an energy-budget model. The blue, black and red curves represent the 10-d rolling mean of the minimal $\left(\Delta \mathrm{T}_{\min }\right)$, average $\left(\Delta \mathrm{T}_{\text {ave }}\right)$ and maximal $\left(\Delta \mathrm{T}_{\max }\right)$ temperature differences, respectively. Approximative leaf flushing date is illustrated by the red vertical line (April $25^{\text {th }}$ ). 


\section{A complex and non-linear response of plant tissue temperature to radiation and wind}

What can we expect if we account for the energy budgets of buds in phenological studies? Unfortunately, the lack of in situ observations for bud temperature does not allow to answer this question. As part of the reflection, we thus applied existing energy balance approaches ${ }^{44,45,49}$ to explore the potential variability in temperature of an isolated bud (Supplementary material). This situation is well representative of the conditions encountered by sun-exposed buds of a tree and especially of deciduous species (i.e. with no or minimum shading). As a first example, we looked at the variability in $\mathrm{T}_{\text {bud }}$ estimated from its energy balance and site meteorological observations for an European Beech forest ${ }^{48}$. On average, $T_{\text {bud }}$ is expected to be higher than $T_{\text {air }}$ during the preseason $\left(\sim 1^{\circ} \mathrm{C}\right.$ in our example; Figure 2b). Day and night $\mathrm{T}_{\text {bud }}$ are higher or lower than $\mathrm{T}_{\text {air }}$ by several degrees. The temperature of buds thus strongly depends on the diurnal radiative cycle and the spectral composition of the light (SW/LW radiation), echoing the observed asymmetrical effect of diurnal temperatures on leaf unfolding ${ }^{38,42,43}$. Applied on four other sites, this approach leads to similar results despite differences in $\mathrm{T}_{\text {bud }}$ profiles induced by differences in radiation along a latitudinal gradient (Supplementary Figure 1). Spring phenology does not only respond to average preseason temperature, but mainly to the accumulated effect of temperature and its dynamics. It is often assumed that chilling and forcing temperature required for budburst are only effective over specific windows, generally between 0 and $5{ }^{\circ} \mathrm{C}$ and over $5^{\circ} \mathrm{C}$, respectively. Daily bud temperature variability might thus be the most important factor influencing leaf unfolding, not necessarily its average temperature. We could expect that the difference in extremum temperature sensed by buds over the preceding months $\left(\Delta \mathrm{T}_{\min }\right.$ and $\Delta \mathrm{T}_{\max }$, Figure $\left.2 \mathrm{~b}\right)$ will inevitably affect the apparent forcing and chilling requirement for leaf unfolding.

Accounting for the energy budget of buds for six common species across Europe (Supplementary Figure 2) we also expect a stronger interannual variability in $\mathrm{T}_{\text {bud }}$ than $\mathrm{T}_{\text {air }}$, as well as different temporal evolutions over the last decades (Figure 3). In our example, buds are expected to warm faster or slower than air depending on location and species, with $20 \%$ and $7 \%$ of the sites exhibiting an increase and a decrease in $\Delta \mathrm{T}$ over 1990-2015, respectively. Even if these trends represent idealized sun-exposed conditions here, we observe that the heterogeneity in $\Delta \mathrm{T}$ evolution results from a complex and non-linear response to the amount of absorbed radiation and convection processes (Supplementary Figure 3). Because leaf unfolding is earlier in 2015 than in 1990, the average amount of absorbed radiation during the preseason slightly decreased over this period, while most of the interannual variability in $\Delta \mathrm{T}$ is driven by conduction and convection (i.e. wind). The difference in air-bud temperature and their non-linear and non-proportional relationship suggests that our current interpretation of the apparent bud sensitivity to 
151

152

153

154

155

156

157

158

159

160

161

162

163

164

165

166

167

168

169

170

171

172

173 a.

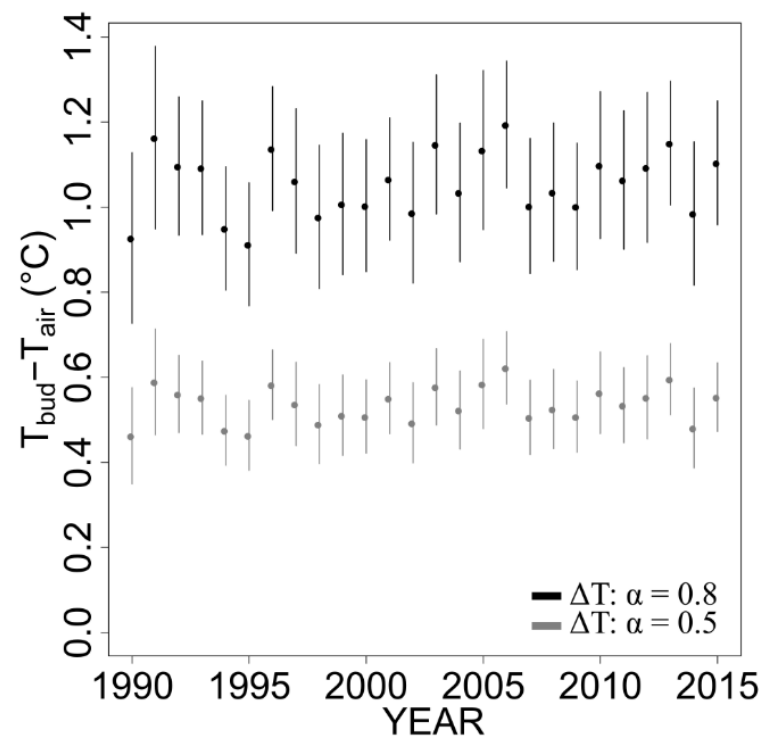

b.

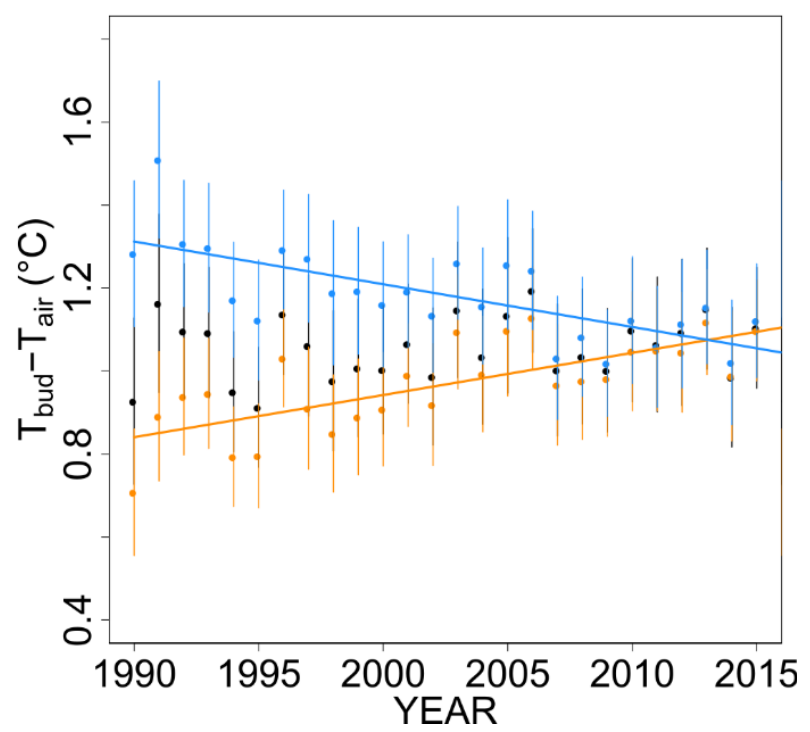

Figure 3 | Potential changes in $\mathbf{T}_{\text {bud }}-\mathbf{T}_{\text {air }}$ over Europe. a. Each point corresponds to the mean $\Delta \mathrm{T}$ simulated for six deciduous species across Europe (1059 sites) under idealized conditions (i.e. sun-exposed buds) using field observation of budburst and global meteorological data (see Supplementary Material). All sites and species were pooled together. Two solar absorptivity values were tested, 0.5 (grey) and 0.8 (black). The error bars represent the spatial and species variability ( $\pm 1 \mathrm{SD}$ around the mean). b. Under these conditions, $\Delta \mathrm{T}$ is expected to decrease (blue) over the period 1990-2016 for 356 sites*species (7\%) and increase for 902 sites*species (orange, 18\%) over a total of 5050 sites*species. The black points correspond to all sites pooled together.

\section{Response to warming will depend on organ properties and local environment}

We illustrated the role of bud energy balance through an idealized and constant representation of buds and their environment for all sites and species. Larger spatial and temporal variations are expected due to the effect of topography, ground albedo (e.g. snow, understory), differences in bud traits (Figure 1b) and micrometeorological conditions ${ }^{50}$ that will affect plant tissues energy balance. By affecting the amount of radiation reaching the buds (Figure 1a), varying ground albedo from 0.1 ( $\sim$ wet bare soil) to $0.9(\sim$ snow) leads to a doubling in preseason $\Delta \mathrm{T}$ (Supplementary Figure 4$)$. Since ground albedo strongly vary in space and over the preseason (e.g snow), we can expect substantial differences in the phenological signal at the regional scale induced by radiation, as already observed from leaf unfolding observations ${ }^{18}$.

Different bud colors or coating will also affect solar absorption of specific wavelengths, while shape and size will modify convection processes and the amount of intercepted radiation (Supplementary Figure 5), and hence, bud temperature. For example, Common Ash (Fraxinus excelsior) has black buds while sycamore (Acer pseudoplatanus) has green buds and mountain ash (Sorbus aucuparia) have dense white trichomes (i.e. hairs) on their surfaces. In our example, a difference in solar absorptivity of 0.3 
174 leads to a doubling in $\Delta \mathrm{T}$ (Figure 2a, Figure 3a). The differences in bud traits can thus partly account for

175 the observed interspecific differences in heat requirement and apparent sensitivity to temperature. This

176 suggests that the phenological response of plants to warming might be more species-specific than we 177 thought, which should be accounted for in large scale studies.

178 Despite its central role at the organ level ${ }^{50,51}$, micrometeorology is rarely accounted for in 179 phenology studies because rarely measured, or simply because it is impossible to account for its effect 180 such as in remote sensing analysis or terrestrial biosphere modelling. Instead, phenology studies, either

181 local or regional, often use meteorological and climate dataset with hourly to daily time resolutions. The 182 use of a steady state energy balance is easily justified under such conditions since thermal time constants 183 of tree buds varies between a few seconds to about ten minutes ${ }^{52}$. Accounting for average preseason 184 radiation and wind conditions might better explain the observed variability in plant phenology than air 185 temperature alone. Here, we only explored spring $\mathrm{T}_{\text {bud }}$ variability in the case of sun-exposed buds with no shading. Accounting for the potential protecting effect of leaves or needles in evergreen species might substantially attenuate the effects of radiation and wind on intra- and bottom-canopy buds. The concomitant use of high-resolution microclimate data and transient energy budget models will be needed to quantify such effects.

\section{Towards a better understanding of the environmental control of plant phenology}

Drivers of phenological events and light are virtually impossible to separate, because daylength and radiation are strongly correlated with the time of year. Accounting for organ energy balances is thus

194 promising for separating the environmental drivers of phenology using a single approach and potentially

195 for reconciliating the differences observed in the field. Applying existing modelling approaches in the context of sun-exposed buds suggested that air temperature might be an imprecise and biased predictor

197 of bud temperature and more importantly of its variability over the months preceding leaf unfolding, which might introduce biases in the analysis of chilling and forcing requirement for budburst. However, we also showed that bud temperature results from a complex combination of several biotic and abiotic factors, and under certain conditions air temperature might remain a good proxy for bud temperature. The examples we have presented demand the reassessment of past results and interpretations that were solely based on air temperature, however current observations do not allow such reassessment. Bud traits and in situ temperature observations are scarcely described in the literature. New experiments and observations are clearly needed for accurately assessing the energy balances of plant organs. Existing studies have mostly focused on leaves, but other organs should also be investigated. Key traits that will 
207 solar absorptivity and heat storage, but organ temperatures (i.e. using thermocouples) concomitant with 208 micrometeorological variables will also need to be directly measured. Because buds do not transpire, 209 their energy budget is simpler than for leaves. Properly calibrated, accounting for bud energy balance 210 could improve the accuracy of phenological models that are still unable to predict the spatiotemporal 211 variability of plant dynamics with satisfactory accuracy ${ }^{53}$.

Finally, we stress that energy balance affects the temperature extrema sensed by plants (Figure 214 2b). The lengthening of the growing season in recent decades has also been associated with an increase 215 in environmental risks. For example, earlier leaf unfolding exposes plants late frost ${ }^{54-56}$ in spring, 216 potentially resulting in dramatic impacts on agriculture ${ }^{57-59}$ and forestry ${ }^{60,61}$. The use of energy balances 217 to study and better predict these environmental risks can provide novel insights into the responses of 218 plants to extreme temperatures and offer more robust predictive tools, which are essential for mitigating 219 the ecological and economic impacts. Temperature of plant organs and their dynamics are still overlooked 220 in both environmental studies and modeling exercises ${ }^{62}$. Energy balance thus plays a key role, not only 221 for plant phenology but also for all other processes since plant tissue temperature will govern key 222 mechanisms such as photosynthesis and respiration and the general functioning of the plant. 


\section{Acknowledgments}

225 The authors would like to acknowledge Dr. Chris Muir and Dr. Renée Marchin Prokopavicius, as well as 226 two anonymous reviewers for their constructive feedbacks on our work. M.P. would like to acknowledge 227 the financial support from the Fonds Wetenschappelijk Onderzoek (FWO; grant no. G018319N) and the 228 H2020 Marie Skłodowska-Curie Actions (LEAF-2-TBM grant no. 891369). J.P. would like to 229 acknowledge the financial support from the European Research Council Synergy grant ERC-SyG-2013230610028 IMBALANCE-P and the Spanish government grant PID2019-110521GB-I00. H.V. 231 acknowledges the support from the European Research Council Starting Grant 637643 232 TREECLIMBERS.

\section{Supplementary information}

235 Already existing models and data were used to support this perspective. This work did not attempt to 236 develop, nor validate, a new model. The full description of the energy budget model (and underlying 237 assumptions) as well as supplementary figures and tables are provided in the Supplementary information. 238 The R code of the model of energy budgets and data sets used to generate the figures and analysis of this 239 manuscript are available from Github: https://github.com/mpeaucelle/Tbud

240 A version of the git repository is archived on Zenodo at https://zenodo.org/record/4173415. 


\section{References}

1. Peñuelas, J. \& Filella, I. Phenology. Responses to a warming world. Science (New York, N.Y.) 294, 793-795; 10.1126/science.1066860 (2001).

2. Peñuelas, J., Rutishauser, T. \& Filella, I. Ecology. Phenology feedbacks on climate change. Science (New York, N.Y.) 324, 887-888; 10.1126/science.1173004 (2009).

3. Ramos-Jiliberto, R., Moisset de Espanés, P., Franco-Cisterna, M., Petanidou, T. \& Vázquez, D. P. Phenology determines the robustness of plant-pollinator networks. Scientific reports 8, 14873; 10.1038/s41598-018-33265-6 (2018).

4. Chuine, I. Why does phenology drive species distribution? Philosophical transactions of the Royal Society of London. Series B, Biological sciences 365, 3149-3160; 10.1098/rstb.2010.0142 (2010).

5. Chmielewski, F.-M. Phenology in Agriculture and Horticulture. In Phenology: An Integrative Environmental Science, edited by M. D. Schwartz. 2nd ed. (Springer Netherlands; Springer ebooks; Imprint: Springer, Dordrecht, 2013), pp. 539-561.

6. Morellato, L. P. C. et al. Linking plant phenology to conservation biology. Biological Conservation 195, 60-72; 10.1016/j.biocon.2015.12.033 (2016).

7. Katelaris, C. H. \& Beggs, P. J. Climate change: allergens and allergic diseases. Internal medicine journal 48, 129-134; 10.1111/imj.13699 (2018).

8. Schwartz, M. D. (ed.). Phenology: An Integrative Environmental Science. 2nd ed. (Springer Netherlands; Springer e-books; Imprint: Springer, Dordrecht, 2013).

9. Delpierre, N. et al. Modelling interannual and spatial variability of leaf senescence for three deciduous tree species in France. Agricultural and Forest Meteorology 149, 938-948; 10.1016/j.agrformet.2008.11.014 (2009).

10. Cleland, E. E., Chuine, I., Menzel, A., Mooney, H. A. \& SCHWARTZ, M. D. Shifting plant phenology in response to global change. Trends in ecology \& evolution 22, 357-365; 10.1016/j.tree.2007.04.003 (2007).

11. Fu, Y. H. et al. Recent spring phenology shifts in western Central Europe based on multiscale observations. Global Ecology and Biogeography 23, 1255-1263; 10.1111/geb.12210 (2014).

12. Jeong, S.-J., HO, C.-H., GIM, H.-J. \& BROWN, M. E. Phenology shifts at start vs. end of growing season in temperate vegetation over the Northern Hemisphere for the period 1982-2008. Global change biology 17, 2385-2399; 10.1111/j.1365-2486.2011.02397.x (2011).

13. Liu, Q. et al. Delayed autumn phenology in the Northern Hemisphere is related to change in both climate and spring phenology. Global change biology 22, 3702-3711; 10.1111/gcb.13311 (2016).

14. Vitasse, Y. et al. Leaf phenology sensitivity to temperature in European trees: Do within-species populations exhibit similar responses? Agricultural and Forest Meteorology 149, 735-744; 10.1016/j.agrformet.2008.10.019 (2009).

15. Wang, S. et al. Temporal Trends and Spatial Variability of Vegetation Phenology over the Northern Hemisphere during 1982-2012. PloS one 11, e0157134; 10.1371/journal.pone.0157134 (2016).

16. Fu, Y. H. et al. Declining global warming effects on the phenology of spring leaf unfolding. Nature 526, 104-107; 10.1038/nature15402 (2015).

17. Huang, M. et al. Velocity of change in vegetation productivity over northern high latitudes. Nature ecology \& evolution 1, 1649-1654; 10.1038/s41559-017-0328-y (2017). 
283

284

285

286

287

288

289

290

291

292

293

294

295

296

297

298

299

300

301

302

303

304

305

306

307

308

309

310

311

312

313

314

315

316

317

318

319

320

321

322

323

324

18. Peaucelle, M. et al. Spatial variance of spring phenology in temperate deciduous forests is constrained by background climatic conditions. Nature communications 10,$5388 ; 10.1038 / \mathrm{s} 41467$ 019-13365-1 (2019).

19. Zohner, C. M., Mo, L., Pugh, T. A. M., Bastin, J.-F. \& Crowther, T. W. Interactive climate factors restrict future increases in spring productivity of temperate and boreal trees. Global change biology; 10.1111/gcb.15098 (2020).

20. Montgomery, R. A., Rice, K. E., Stefanski, A., Rich, R. L. \& Reich, P. B. Phenological responses of temperate and boreal trees to warming depend on ambient spring temperatures, leaf habit, and geographic range. Proceedings of the National Academy of Sciences of the United States of America 117, 10397-10405; 10.1073/pnas.1917508117 (2020).

21.Zohner, C. M., Benito, B. M., Svenning, J.-C. \& Renner, S. S. Day length unlikely to constrain climate-driven shifts in leaf-out times of northern woody plants. Nature Clim Change 6, 1120-1123; 10.1038/nclimate3138 (2016).

22. Peñuelas, J. et al. Complex spatiotemporal phenological shifts as a response to rainfall changes. New Phytologist 161, 837-846; 10.1111/j.1469-8137.2004.01003.x (2004).

23. Papagiannopoulou, C. et al. Vegetation anomalies caused by antecedent precipitation in most of the world. Environ. Res. Lett. 12, 74016; 10.1088/1748-9326/aa7145 (2017).

24. Fu, Y. H. et al. Nutrient availability alters the correlation between spring leaf-out and autumn leaf senescence dates. Tree physiology 39, 1277-1284; 10.1093/treephys/tpz041 (2019).

25. Seyednasrollah, B., Swenson, J. J., Domec, J.-C. \& Clark, J. S. Leaf phenology paradox: Why warming matters most where it is already warm. Remote Sensing of Environment 209, 446-455; 10.1016/j.rse.2018.02.059 (2018).

26. Chuine, I., Morin, X. \& Bugmann, H. Warming, photoperiods, and tree phenology. Science (New York, N.Y.) 329, 277-8; author reply 278; 10.1126/science.329.5989.277-e (2010).

27. Vitasse, Y. \& Basler, D. What role for photoperiod in the bud burst phenology of European beech. Eur J Forest Res 132, 1-8; 10.1007/s10342-012-0661-2 (2013).

28. Way, D. A. \& Montgomery, R. A. Photoperiod constraints on tree phenology, performance and migration in a warming world. Plant, cell \& environment 38, 1725-1736; 10.1111/pce.12431 (2015).

29. Caffarra, A., Donnelly, A. \& Chuine, I. Modelling the timing of Betula pubescens budburst. II. Integrating complex effects of photoperiod into process-based models. Clim. Res. 46, 159-170; $10.3354 / \mathrm{cr} 00983$ (2011).

30. Körner, C. \& Basler, D. Plant science. Phenology under global warming. Science (New York, N.Y.) 327, 1461-1462; 10.1126/science.1186473 (2010).

31. Fu, Y. H. et al. Daylength helps temperate deciduous trees to leaf-out at the optimal time. Global change biology 25, 2410-2418; 10.1111/gcb.14633 (2019).

32. Singh, R. K., Svystun, T., AlDahmash, B., Jönsson, A. M. \& Bhalerao, R. P. Photoperiod- and temperature-mediated control of phenology in trees - a molecular perspective. New Phytologist 213, 511-524; 10.1111/nph.14346 (2017).

33. Flynn, D. F. B. \& Wolkovich, E. M. Temperature and photoperiod drive spring phenology across all species in a temperate forest community. New Phytologist 219, 1353-1362; 10.1111/nph.15232 (2018). 
34. Brelsford, C. C., Nybakken, L., Kotilainen, T. K. \& Robson, T. M. The influence of spectral composition on spring and autumn phenology in trees. Tree physiology 39, 925-950; 10.1093/treephys/tpz026 (2019).

35. Strømme, C. B. et al. UV-B and temperature enhancement affect spring and autumn phenology in Populus tremula. Plant, cell \& environment 38, 867-877; 10.1111/pce.12338 (2015).

36. Fu, Y. H. et al. Increased heat requirement for leaf flushing in temperate woody species over 19802012: effects of chilling, precipitation and insolation. Global change biology 21, 2687-2697; 10.1111/gcb.12863 (2015).

37. Huang, Y., Jiang, N., Shen, M. \& Guo, L. Effect of preseason diurnal temperature range on the start of vegetation growing season in the Northern Hemisphere. Ecological Indicators 112, 106161; 10.1016/j.ecolind.2020.106161 (2020).

38. Meng, F. et al. Opposite effects of winter day and night temperature changes on early phenophases. Ecology 100, e02775; 10.1002/ecy.2775 (2019).

39. Zhang, S., Isabel, N., Huang, J.-G., Ren, H. \& Rossi, S. Responses of bud-break phenology to daily-asymmetric warming: daytime warming intensifies the advancement of bud break. International journal of biometeorology 63, 1631-1640; 10.1007/s00484-019-01776-0 (2019).

40. Meng, L. et al. Divergent responses of spring phenology to daytime and nighttime warming. Agricultural and Forest Meteorology 281, 107832; 10.1016/j.agrformet.2019.107832 (2020).

41. Bigler, C. \& Vitasse, Y. Daily Maximum Temperatures Induce Lagged Effects on Leaf Unfolding in Temperate Woody Species Across Large Elevational Gradients. Frontiers in plant science 10, 398; 10.3389/fpls.2019.00398 (2019).

42. Fu, Y. H. et al. Three times greater weight of daytime than of night-time temperature on leaf unfolding phenology in temperate trees. The New phytologist 212, 590-597; 10.1111/nph.14073 (2016).

43. Piao, S. et al. Leaf onset in the northern hemisphere triggered by daytime temperature. Nature communications 6, 6911; 10.1038/ncomms7911 (2015).

44. HAMER, P. The heat balance of apple buds and blossoms. Part I. Heat transfer in the outdoor environment. Agricultural and Forest Meteorology 35, 339-352; 10.1016/0168-1923(85)90094-2 (1985).

45. Landsberg, J. J., Butler, D. R. \& Thorpe, M. R. Apple bud and blossom temperatures. Journal of Horticultural Science 49, 227-239; 10.1080/00221589.1974.11514574 (1974).

46. Grace, J. The temperature of buds may be higher than you thought. New Phytologist 170, 1-3; 10.1111/j.1469-8137.2006.01675.x (2006).

47. Jones, H. G. Plants and microclimate. A quantitative approach to environmental plant physiology (Cambridge university press, Cambridge, 2013).

48. Granier, A., Bréda, N., Longdoz, B., Gross, P. \& Ngao, J. Ten years of fluxes and stand growth in a young beech forest at Hesse, North-eastern France. Ann. For. Sci. 65, 704; 10.1051/forest:2008052 (2008).

49. Muir, C. D. tealeaves: an R package for modelling leaf temperature using energy budgets. $A o B$ PLANTS 11, plz054; 10.1093/aobpla/plz054 (2019).

50. Zellweger, F. et al. Forest microclimate dynamics drive plant responses to warming. Science (New York, N.Y.) 368, 772-775; 10.1126/science.aba6880 (2020). 
51. Bailey, B. N., Stoll, R., Pardyjak, E. R. \& Miller, N. E. A new three-dimensional energy balance model for complex plant canopy geometries: Model development and improved validation strategies. Agricultural and Forest Meteorology 218-219, 146-160; 10.1016/j.agrformet.2015.11.021 (2016).

52. Michaletz, S. T. \& Johnson, E. A. A heat transfer model of crown scorch in forest fires. Can. J. For. Res. 36, 2839-2851; 10.1139/x06-158 (2006).

53. Richardson, A. D. et al. Terrestrial biosphere models need better representation of vegetation phenology: results from the North American Carbon Program Site Synthesis. Glob Change Biol 18, 566-584; 10.1111/j.1365-2486.2011.02562.x (2012).

54. Liu, Q. et al. Extension of the growing season increases vegetation exposure to frost. Nature communications 9, 426; 10.1038/s41467-017-02690-y (2018).

55. Ma, Q., Huang, J.-G., Hänninen, H. \& Berninger, F. Divergent trends in the risk of spring frost damage to trees in Europe with recent warming. Global change biology 25, 351-360; $10.1111 / \mathrm{gcb} .14479$ (2019).

56.Zohner, C. M. et al. Late-spring frost risk between 1959 and 2017 decreased in North America but increased in Europe and Asia. Proceedings of the National Academy of Sciences of the United States of America; 10.1073/pnas.1920816117 (2020).

57. Xiao, L. et al. Estimating spring frost and its impact on yield across winter wheat in China. Agricultural and Forest Meteorology 260-261, 154-164; 10.1016/j.agrformet.2018.06.006 (2018).

58. Unterberger, C. et al. Spring frost risk for regional apple production under a warmer climate. PloS one 13, e200201; 10.1371/journal.pone.0200201 (2018).

59. Leolini, L. et al. Late spring frost impacts on future grapevine distribution in Europe. Field Crops Research 222, 197-208; 10.1016/j.fcr.2017.11.018 (2018).

60. Greco, S. et al. Late Spring Frost in Mediterranean Beech Forests: Extended Crown Dieback and Short-Term Effects on Moth Communities. Forests 9, 388; 10.3390/f9070388 (2018).

61. Augspurger, C. K. Spring 2007 warmth and frost: phenology, damage and refoliation in a temperate deciduous forest. Functional Ecology 23, 1031-1039; 10.1111/j.1365-2435.2009.01587.x (2009).

62. Dong, N., Prentice, I. C., Harrison, S. P., Song, Q. H. \& Zhang, Y. P. Biophysical homoeostasis of leaf temperature: A neglected process for vegetation and land-surface modelling. Global Ecol Biogeogr 26, 998-1007; 10.1111/geb.12614 (2017). 